\title{
Research Article \\ Porosity Distribution in Composite Structures with Infrared Thermography
}

\author{
Cinzia Toscano, ${ }^{1,2}$ Carosena Meola, ${ }^{2}$ and Giovanni Maria Carlomagno ${ }^{2}$ \\ ${ }^{1}$ Italian Aerospace Research Centre (CIRA), Via Maiorise, 81043 Capua, Italy \\ ${ }^{2}$ Department of Industrial Engineering, Aerospace Section, University of Naples Federico II, Via Claudio 21, 80125 Napoli, Italy
}

Correspondence should be addressed to Carosena Meola; carmeola@unina.it

Received 14 January 2013; Revised 14 March 2013; Accepted 17 March 2013

Academic Editor: Harry Siu-lung Ku

Copyright (c) 2013 Cinzia Toscano et al. This is an open access article distributed under the Creative Commons Attribution License, which permits unrestricted use, distribution, and reproduction in any medium, provided the original work is properly cited.

\begin{abstract}
Composite structures are increasingly used in the transport industry especially in the aeronautical sector thanks to their favorable strength-to-weight ratio with respect to metals. However, this is true if the final part is defects free and complies with quality requirements. A main weakness in composites is porosity, which is likely to be introduced during manufacturing processes and which may knock down the material characteristics affecting its performance in service. Porosity plays a key role in sandwich structures, which involve novel metal foams as core, since the foam performance strongly depends on size and distribution of pores. The determination of porosity is mostly attained by destructive methods, which supply only a general indication linked to the production part number. Conversely, composites may entail local significant variation of porosity, which may be discovered only with effective nondestructive techniques. The attention of the present work is focused on the possibility to use infrared thermography to get information about the amount and distribution of porosity. In particular, two techniques: flash thermography and lock-in thermography are used to comply with requirements of both monolithic composites and metal foams.
\end{abstract}

\section{Introduction}

Composite structures are increasingly used in the transport industry especially in the construction of aircraft [1] thanks to their favorable strength-to-weight ratio with respect to metals. Adversely, one of the main problems is related to their intrinsic inhomogeneous structure and to defects that can be inadvertently induced during their manufacture.

The mostly used composites include a polymeric matrix reinforced with either carbon, or glass fibers, which are generally referred to as fiber reinforced polymers (FRPs). Generally, plies of fibers impregnated with resin are overlaid owing to a fixed stacking sequence and cured in autoclave. The autoclave cycle involves the combined effects of temperature and pressure. Temperature is needed to activate and to control the chemical reactions in the resin, while pressure may squeeze off the resin in excess to consolidate the stacked plies and to minimize the amount of entrapped gas between the plies and within the resin [2]. In particular, setting up the vacuum pressure and maintaining it for a fixed interval have been individuated as critical parameters to be carefully monitored $[3,4]$ to avoid undesired formation of voids in the laminate. In fact, the presence of porosity can reduce the interlaminar shear strength causing delamination (interlamina debonding). On the other side, the presence of regions of fibers unsupported by the matrix can induce local stress concentration, with consequent severe degradation of strength and stiffness in-service. That is why the porosity level must be known and taken into account.

Many of the aircraft parts are made of sandwich structures, which include a thick but lightweight core between two thin yet stiff skins. Sandwich structures may be affected by different types of defects involving the characteristic defects of the core and of the skin materials, as well as debonding at the interface skin-core. The materials mostly used as skins are composites like fiber reinforced polymers. The core may be made of different types of cellular foams, honeycombs, balsa wood, and so forth. Metal foams are very attractive because they fill the desired volume with a material which somewhat maintains mechanical characteristics similar to 
the initial solid material, but includes less metal with significant reduction in weight. Therefore, they have good stiffness and strength-to-weight ratios as well as the ability to absorb impact energy and electromagnetic waves. In addition, the air pockets that are contained in the structure confer good acoustic damping, thermal insulation and allow retaining noncombustibility features. The quality of a foam requires two many factors to be accomplished which are a uniform distribution of pores and pores not exceeding a certain size.

Thus, for the performance of a component, whatever the material (a composite laminate or a sandwich core foam), the amount and, above all, the distribution of porosity, as well as the size of pores, are very important.

Many techniques have been investigated for estimation of porosity in fiber reinforced composites [5-13]. Some are destructive like the acid digestion $[5,10]$, which can supply information only by statistical inference and so has to be regarded as a reference rather than a quality assessment method. The most commonly accepted method is the ultrasonic (US) one for which the porosity percentage is linearly correlated to the attenuation of ultrasonic waves [510]; however, such an attenuation is affected by size, shape, and orientation of the pores leading to significant errors in quantitative data.

Therefore, the attention has been devised also towards other methods such as computed radiography $[6,12]$ and infrared thermography $[11,13]$. Computed radiography (CT) [12] may be used to overcome some of the US limitation regarding size, shape, and position of all individual pores. Adversely, it involves difficulties in testing real parts (it is mainly suitable for small samples) as well as safety at work concerns.

A methodology which acts in nondestructive and completely noninvasive way and which does not involve any specific surface prerequisites, nor safety at work concerns, is infrared thermography. It has proved its suitability, through evaluation of porosity percentages linked to thermal diffusivity measurements mainly in monolithic composites [11, 13].

Conversely, there is very poor literature concerned with porosity of metal foams; this is because many of the nondestructive methods today available are not applicable to porous media [14] or are difficult to use and not really effective. In fact, ultrasonic attenuation is mostly effective for waterproof surfaces. On the other hand, air-coupled inspection is not appropriate due to the extremely high acoustic impedance of metals and the corresponding poor transmission coefficients for sound from air. Information about porosity may be derived from elastic properties obtained through laser-ultrasound probing $[15,16]$, but this seems limited to specimens. The better technique for resolving individual pores remains again CT, but its applicability seems limited to samples, while industrial production is demanding for proper inline control. A possible candidate to assess porosity distribution in large panels may be lock-in thermography owing to a recent investigation performed by Carlomagno et al. [17] on an aluminum foam.

The attention of the present work is focused on the suitability of infrared thermography as nondestructive technique to be used for porosity evaluation of both monolithic composites and novel metal foams used as core in sandwich structures. Two purposes are pursued. One is to add new data in terms of thermal diffusivity, evaluated with flash thermography, correlated to porosity in a carbon reinforced polymer involving variation of stacking sequence and of curing pressure. The other one is to demonstrate the usefulness of lock-in thermography in the evaluation of porosity distribution in an aluminum foam.

\section{Basic Thermographic Methods for Nondestructive Inspection}

There are two basic thermographic techniques, which can be used for nondestructive inspection: pulse thermography (PT) and lock-in thermography (LT). Other techniques such as pulse phase (PPT), time-resolved-infrared (TRIR), and vibrothermography can be arranged which are variations of PT and LT because they include a different heating method or a different processing algorithm $[18,19]$.

2.1. Pulse Thermography. PT consists simply in the stimulation of the object (under evaluation) by a heating pulse and monitoring of its surface temperature variation during the transient heating, or cooling, phase. Heating is generally performed by lamps, flash lamps, scanning lasers, or hot air jets. Cooling can be practically attained mainly by cold air jets. Of course, air jets (hot or cold) can be used only on a massive surface since jet impingement may damage delicate artworks.

For the case of slabs, analysis with PT can be performed in two different modes: transmission and reflection. In the first mode, the infrared camera views the rear face, that is, the face opposite to the heating/cooling source; in the second one, both heating (cooling) source and camera are positioned on the same side. However, the latter is most commonly applied since only one side of the object under test is frequently available. The thermal energy propagates by conduction inside the material while the infrared camera monitors the temperature variation over the viewed surface. Obviously, for a uniform heating of the surface and homogeneous material, the temperature $T$ distribution is uniform; the presence of a defect at a certain depth interferes with the heat flow causing local surface temperature variations.

The visibility of the defect can be evaluated by the parameter $D_{T}[20]$ :

$$
D_{T}=\frac{|\Delta T|}{\left|\Delta T_{s}\right|}=\frac{\left|T_{s}-T_{d}\right|}{\left|T_{s}-T_{r}\right|},
$$

where $T_{d}$ is the temperature over a defective zone, $T_{s}$ is the temperature in a sound zone, and $T_{r}$ is a reference temperature. More specifically, $T_{r}$ is the temperature of the sound material before starting of transient heating, or cooling $T_{r}=T_{s(t=0)}$ (i.e., the temperature of the first thermal image taken at $t=0 \mathrm{~s}$ in the time sequence). The dimensionless temperature difference $D_{T}$ in (1) can assume either positive, or negative values, the sign depending on the test procedure 
(acquisition during heating, or during cooling) and on the thermal properties of both the sound material and the material of which the defect is made.

Generally, a deep defect becomes visible later than a shallow one and a larger defect produces a more marked temperature difference. Thus, a complete (from surface to depth) evaluation of a material is performed by acquiring thermal images (also called thermograms) in a time sequence. The thermal image may be shown in grey, or color levels (10, $16, \ldots, 112$, or more) on the computer monitor, each level being related to a temperature interval; such interval depends on the selected range (the minimum allowable range for the scanner is often about 2 degrees). Information about the defect parameters such as size, depth, and thermal resistance can be obtained by applying postprocessing procedures to the thermograms.

The defect detection is limited by the camera Signal-toNoise Ratio (SNR) or by the Noise Equivalent Temperature Difference (NETD). In addition, the surface finish is of great importance since variations in surface roughness, cleanliness, uniformity of paint, and other surface conditions can cause variations in the emissivity coefficient and affect the temperature measurement. These aspects were analyzed by Meola and Carlomagno [20, 21].

2.2. Lock-In Thermography. The concept of lock-in thermography was first introduced by Carlomagno and Berardi [22] and later further investigated by other researchers [23-30]. Classical lock-in thermography is basically performed with a modulated heating lamp and is called optical lock-in thermography and abbreviated as LT or OLT (some authors also call it modulated thermography MT). The energy, generated by halogen lamps, is delivered to the object surface in the form of periodic thermal waves. The thermographic system is coherently coupled to the thermal wave source which is operated in such a way that a sinusoidal temperature modulation results. This modulation is obtained from a nonlinear electrical signal produced by the lock-in module which allows also for frequency variation. The system collects a series of images and compares the modulated heating to the measured temperatures by extracting the sinusoidal wave pattern at each point of the image. In a homogeneous material, the basic relationship relates the thermal diffusion length $\mu$ to the thermal diffusivity coefficient $\alpha$ and to the wave frequency $f=\omega / 2 \pi$ :

$$
\mu=\sqrt{\frac{\alpha}{\pi f}} .
$$

In the classical optical lock-in method both lamp and camera are located on the same specimen side. The thermal wave hits the material surface, propagates inside it, and gets reflected by local in-homogeneities (i.e., parts where the heat propagation parameters change). The reflected wave interferes with the surface wave producing an oscillating interference pattern, which can be measured in terms of amplitude $A(z)$, or phase angle $\phi(z)$, that, respectively, produces amplitude, or phase, images. The depth range for the amplitude image is given by $\mu$ while the maximum depth $p$, which can be reached for the phase image, corresponds to $1.8 \mu$.

Therefore, the material thickness, which can be inspected, depends on the wave period (the longer the period, the deeper the penetration) and on the thermal material properties (thermal conductivity and volumetric specific heat). Generally, tests start at a quite high wave frequency, at which only surface (or low depth) defects are visible, and later on, to inspect deeper layers, the frequency is decreased until the entire thickness has been traversed, or the minimum selectable frequency has been reached. Results are generally presented as phase images where a local variation of color indicates a local variation of phase angle $\phi$ and, in turn, a local variation of material thermal properties. The images may be stored for successive analysis to acquire information about size, depth, and nature of defects.

\section{The Addressed Problems}

The attention is devoted to the evaluation of porosity distribution in both monolithic composite materials and sandwich metal foams cores. For the first topic, a carbon reinforced polymer is considered with porosity induced through alteration of the autoclave curing pressure. With regard to the second topic, the aim is to gain information about the cellular structure of an aluminum foam to be used as novel core material in sandwich structures.

Tests are carried out using the SC5500 infrared camera by Flir systems, which is a stirling cooled focal plane array camera equipped with InSb detector working in the medium $3-5 \mu \mathrm{m}$ wavelength range and of spatial resolution $320 \times 256$ pixels.

3.1. Carbon Fiber Reinforced Polymers. Several coupons are fabricated by hand laying-up 24 plies with stacking sequence $\left[45^{\circ} /-45^{\circ}\right]_{\mathrm{s}}$; then, the created $10 \times 5 \mathrm{~cm}^{2}$ stacks are put in a vacuum bag and processed in autoclave at a fixed temperature-pressure curing cycle. Of course, to obtain a good material, the pressure has to be set according to the indications supplied by the material provider. Instead, to induce porosity formation, in some of the laminates, the applied pressure is set to be only a percentage of the prescribed one (7 bar gauge). More specifically, coupons are collected into five groups each cured at a different pressure percentage, being, respectively, $100 \%, 75 \%, 50 \%, 25 \%$, and $0 \%$. In addition, two thin (thickness $0.06 \mathrm{~mm}$ ) kapton disks, $20 \mathrm{~mm}$ in diameter, are overlapped and inserted in the middle of the stacking sequence in one half part of each coupon to simulate local delamination. In this way, the same coupon is used to ascertain the capability of infrared thermography to deal with two types of problems: evaluate the porosity amount and distribution, detect thin delaminations, and slag inclusions.

The resulting porosity for each coupon's group is calculated using the law of mixtures and by knowing the measured average density of the coupons [31]. Specifically, from each type of coupon, 3 small pieces are cut (destructive method) and weighted by a digital balance to finally know, through 


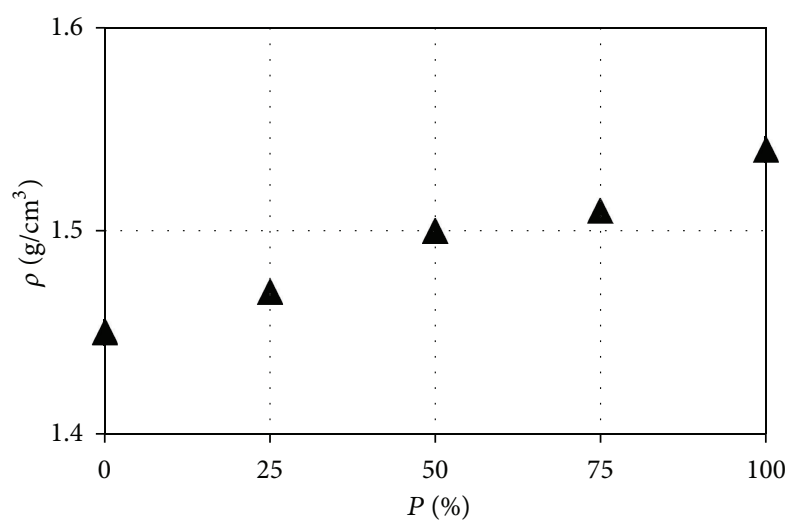

FIgURE 1: Variation of density with the curing pressure.

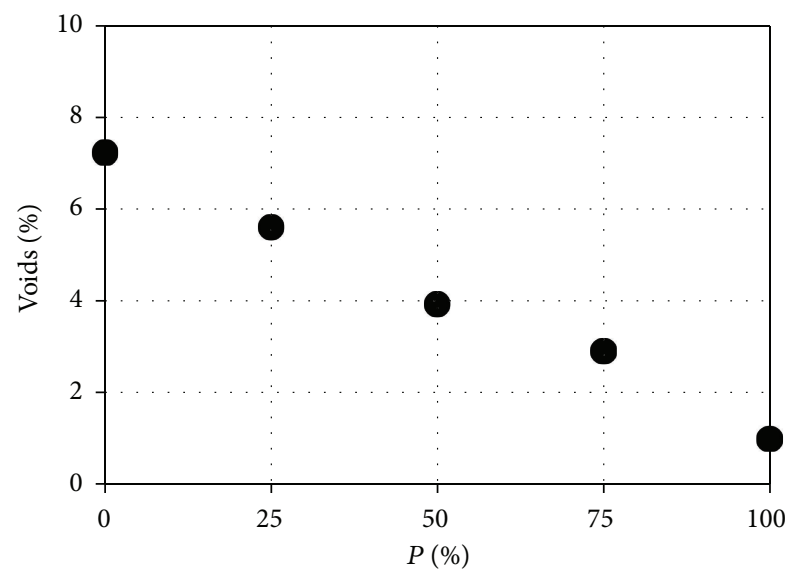

FIgURE 2: Percentage of voids with varying the curing pressure.

the Archimedes principle, their density $\rho$. The law of mixtures gives

$$
\rho=\frac{\rho_{f} V_{f}+\rho_{r} V_{r}}{V_{f}+V_{r}}
$$

with $V_{f}$ and $V_{r}$, respectively, volume of fibers and of resin, whereas $\rho_{f}$ and $\rho_{r}$ are the density of the fibers and of the resin ( $\rho_{f}$ and $\rho_{r}$ are provided by the prepreg lamina supplier). Equation (3) may be rewritten by introducing the volume of voids $V_{v}$ :

$$
\rho=\frac{\rho_{f} V_{f}+\rho_{r} V_{r}}{V_{f}+V_{r}+V_{v}}
$$

Being $V_{f}+V_{r}+V_{v}$ the actual total volume of the piece, from (3) and (4) it is easy to calculate $V_{f} / V_{T}$ and $V_{r} / V_{T}$ and so the porosity volume fraction $V_{v} / V_{T}$. Plots of density and fraction of voids expressed as volume percentages against the curing pressure are shown in Figures 1 and 2, respectively. As can be seen, there is a linear increase of density and decrease of voids formation by increasing the curing pressure.

Then, each coupon is tested using PT in transmission through the flash method [32]. Just to supply some hints about this method, under the assumption of adiabatic conditions and instantaneous pulse heating of intensity $Q_{i}$, the temperature on the rear face of a sample is obtained by solving the one dimensional thermal conduction equation for ideal conditions (i.e., no losses):

$$
T(z, t)-T_{i}=\frac{Q_{i}}{\rho c s}\left[1+2 \sum_{n=1}^{\infty}(-1)^{n} \exp \left(\frac{-n^{2} \pi^{2}}{z^{2}} \alpha t\right)\right]
$$

$s$ being the sample thickness, $\rho$ the density, $c$ the specific heat, $T_{i}$ the initial constant temperature, and $t$ the time after pulse heating. The thermal diffusivity $\alpha$ is determined from the relationship:

$$
\alpha=\frac{0.138 s^{2}}{t_{1 / 2}}
$$

with $t_{1 / 2}$ the time for the sample's backside temperature rise to reach half of its maximum $T_{M / 2}$ value as shown in Figure 3. Of course, this method [32] was first conceived for temperature measurements with contact transducers (e.g., leaf thermocouples); while now, it is being used in combination with infrared thermography. The usefulness of the infrared camera is obvious since it allows for fast monitoring of temperature variations and for evaluation of the thermal diffusivity pixel by pixel over a given, ever large, area. The possibility of mapping the thermal diffusivity is an advantage in view of accounting for local variations due to presence of local material in-homogeneities.

In this work, an Hensel Flash head and power supply, able to emit 6000 Joules in $1 / 400 \mathrm{~s}$, is used to instantaneously heat up the inspected coupon [33]. The temperature-time variation on the opposite side of the coupon is monitored by the infrared camera.

Through analysis of the images with an ad hoc developed Matlab tool and by applying (6), the average thermal diffusivity through the coupon thickness is evaluated point by point over the entire surface of each coupon.

Two maps are shown in Figures 4 and 5, respectively, for coupons A and B, respectively, cured at $100 \%$ and $0 \%$ of the prescribed curing pressure. The location of the simulated delamination is highlighted by a red circle. In both figures is also indicated with a yellow rectangle the area where the average thermal diffusivity is calculated.

The average $\alpha$ values are plotted against the porosity percentage in Figure 6. It is worth noting that the highest value of the thermal diffusivity is that measured over a specimen cured at $P=100 \%$ that should mean $0 \%$ porosity; in reality, the porosity level is not zero but $0.93 \%$ as ascertained through the volumetric method. As can be seen, on the overall, the increasing of porosity percentage entails a correspondent reduction of the average diffusivity. Thus, it is possible from the distribution of the thermal diffusivity to gain information about the distribution of porosity. More exactly, from the nondestructively obtained thermal diffusivity map, it is possible to gain information on the porosity distribution over the whole sample; whereas, on the contrary, the measure of density through the destructive volumetric method is just a local action regarding only a very small part of the sample. 


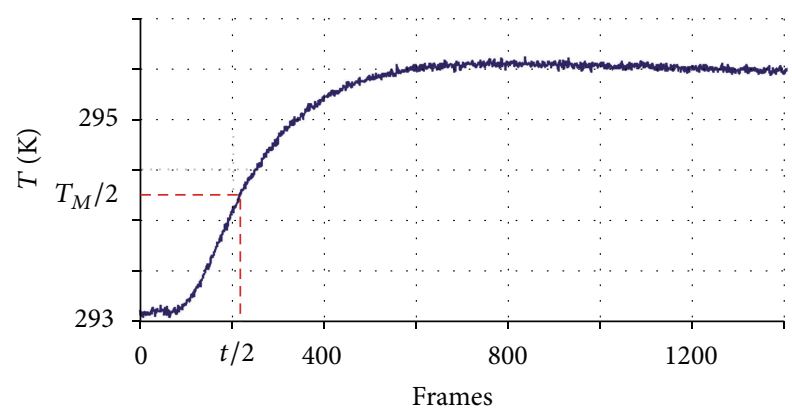

FIgURE 3: Typical temperature plot.

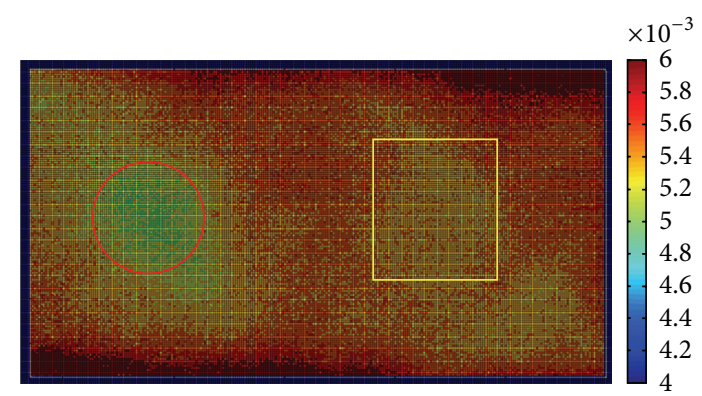

FIgURE 4: Diffusivity map of the coupon A cured at 100\% pressure with $0.93 \%$ of porosity.

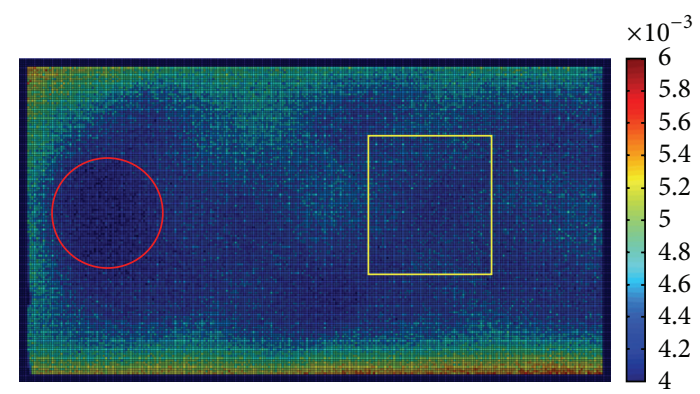

Figure 5: Diffusivity map of the coupon B cured at $0 \%$ pressure with $6 \%$ of porosity.

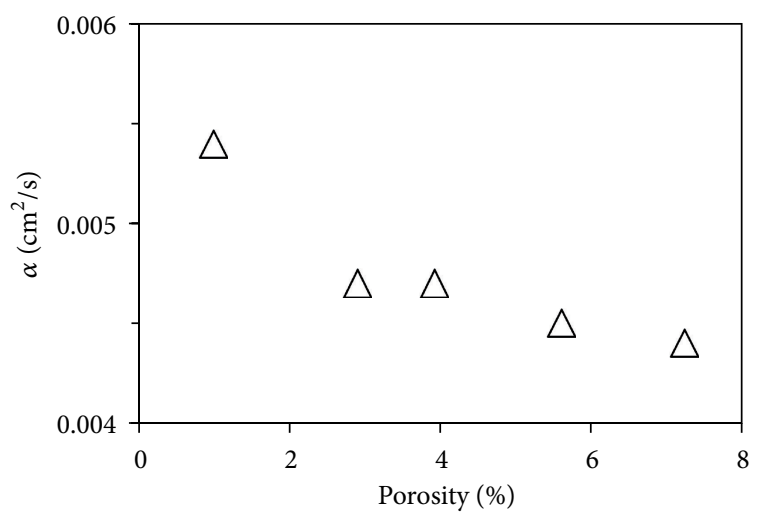

FIGURE 6: Thermal diffusivity against porosity for CFRP.
For a quantitative analysis, by calling $\Delta \varsigma$ and $\Delta \alpha$ the variation of porosity and thermal diffusivity, respectively, we can introduce a parameter $\psi$ defined as

$$
\psi=\frac{\Delta \alpha}{\Delta \varsigma}
$$

which can also be regarded as a resolution measure of the employed method. It is possible to see that $\psi$ attains first a value of 5 until a porosity level of $4 \%$, while it drops below 2 as the porosity increases towards $8 \%$.

The obtained results are also compared to those present in the literature [13]. As a first comment, the composite herein used has thermal diffusivity of about $0.0054 \mathrm{~cm}^{2} / \mathrm{s}$ (for $P=$ $100 \%)$ which is higher than that of the material used in [13] (about $0.004 \mathrm{~cm}^{2} / \mathrm{s}$ ). In addition, there is a general agreement regarding the $\psi$ trend. In fact, data of [13] displays first a lower value $\psi=3.75$ for porosity in the range $0-4 \%$, but with a value of 3 for porosity between 0 and $10 \%$, which is close to the average value attained by present data. It has to be noted that the agreement is with experimental data of [13] in transmission mode. Indeed, this is a valuable result in favor of pulse thermography to be used to assess porosity variations as linked to the variation of thermal diffusivity.

Of course, more tests are necessary to completely assess the validity of the $\psi$ parameter. Conversely, it is not possible to establish a direct correlation between the thermal diffusivity and the level of porosity. This because the thermal diffusivity, for a given composite such as carbon/epoxy, depends on many factors (type of carbon fiber and matrix, stacking sequence, etc.) and not only and simply on the porosity percentage.

3.2. Metal Foams. The today energy-saving imperative has led the aircraft industry to searching for novel materials which must be light and stiff enough at the same time since a reduction in fuel consumption is directly linked to weight reduction. The metal foams are a fairly new class of materials, which can be obtained by compact melting of metal alloy powders, typically aluminum or titanium. An aluminum foam specimen is shown in Figure 7. The main characteristic of this material is porosity which can reach almost the 75$80 \%$ of the total volume (Figure $7(\mathrm{~b})$ ). It is largely used to increase sound dampening and flames resistance; moreover, it is characterized by high stiffness and a very good resistance to impacts [14].

The use of lock-in thermography in the evaluation of aluminum foams was recently investigated; the obtained results were compared with those coming from the most commonly used volumetric method and a good agreement was found [17]. Some phase images of an aluminum foam plate $28 \mathrm{~cm}$ long, $8 \mathrm{~cm}$ wide and $3 \mathrm{~cm}$ thick, and of average density $0.78 \mathrm{~g} / \mathrm{cm}^{3}$ are shown in Figure 8 . As can be seen, with decreasing the heating frequency $f$, the inner porosity cluster (encircled in Figures 8(a)-8(d)) spatial distribution can be highlighted. In addition, it is possible to find out the presence of pores above a certain fixed size, which represents a great advantage considering that, as already affirmed, it 


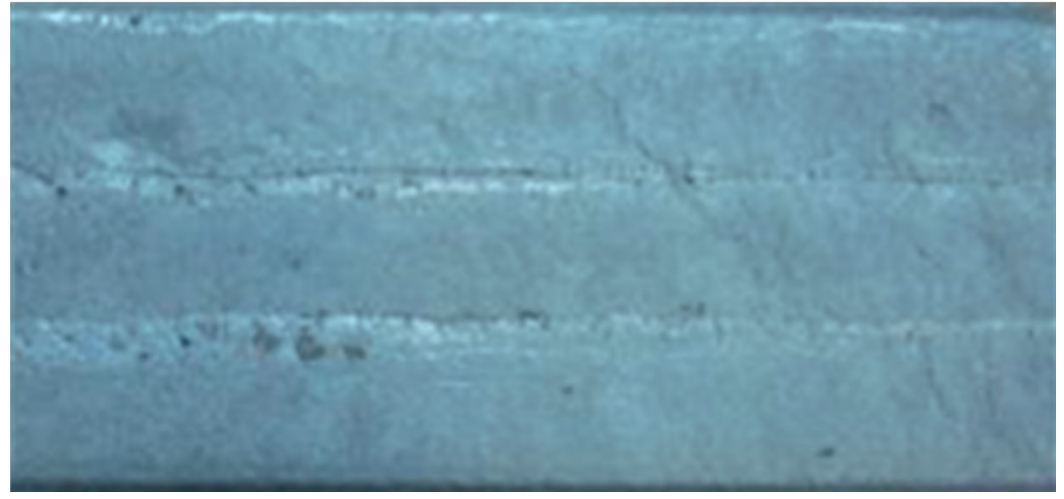

(a) Front side surface finish

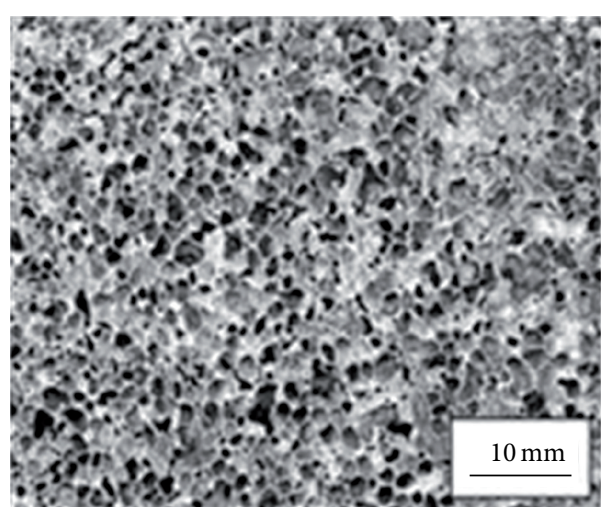

(b) Cut view

Figure 7: An aluminum foam specimen.
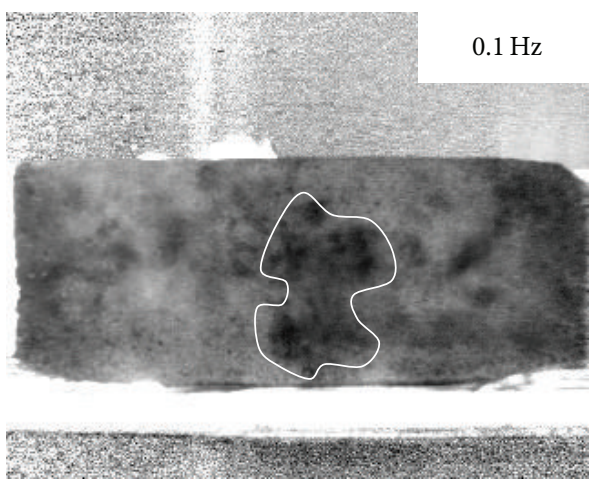

(a)

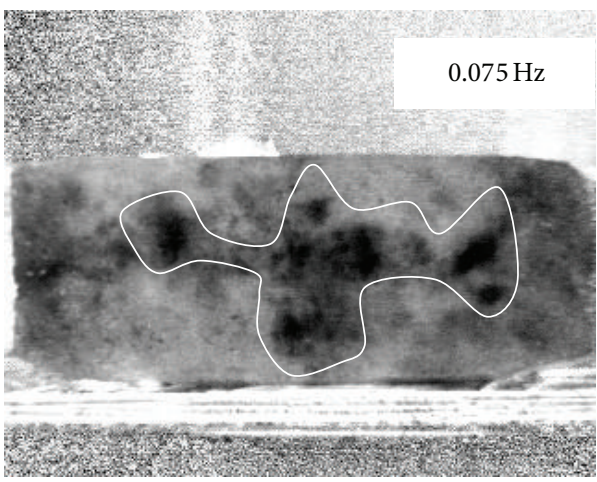

(c)

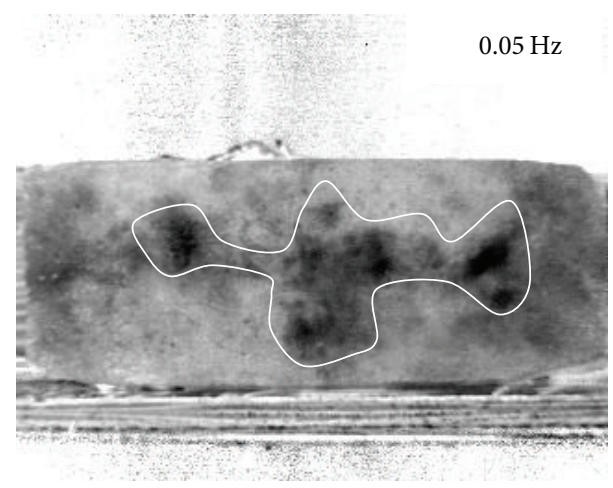

(b)

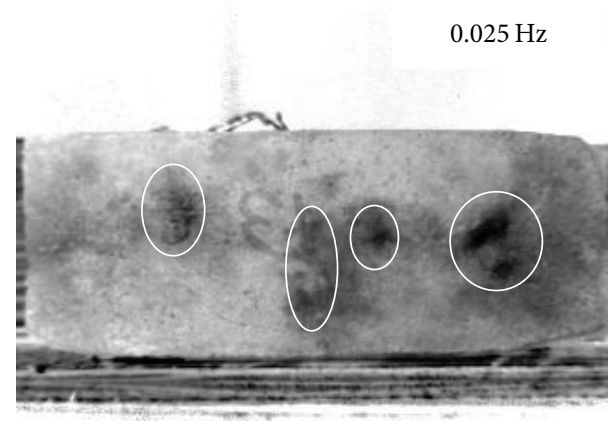

(d)

Figure 8: Phase images of an aluminum foam slab, taken by varying the heating frequency.

is more important having control of size and distribution of pores rather than knowing the mean density value. A demonstration of this is given in Figure 9. In fact, the phase image taken at $f=0.04 \mathrm{~Hz}$ (Figure 9(b)) displays a big pore (encircled), which is located in the zone that was encircled over the sample (Figure $9(\mathrm{c})$ ). Such a zone was cut to obtain a small specimen; Figure $9(\mathrm{~d})$ shows a cut view of such a specimen, which confirms the presence of the big pore detected by lock-in thermography (Figure 9(b)). In addition, it has to be observed that such a pore is not present on the phase image taken at $f=0.5 \mathrm{~Hz}$ (Figure 9(a)) while it appears for $f=0.04 \mathrm{~Hz}$ (Figure 9(b)) because it is located at a certain depth from the upper surface (Figure 9(c)).

These results bear witness for the capability of lock-in thermography in the evaluation of metal foams performance; of course, by knowing the spatial resolution (pixels/mm), it is possible to evaluate the size of pores at different layers through the material thickness. 


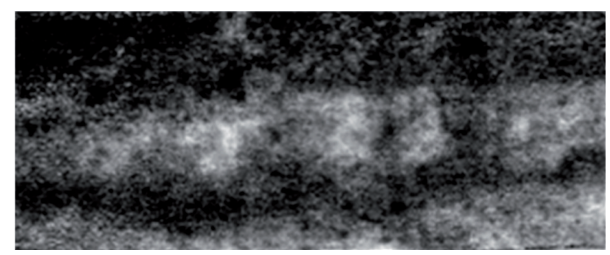

(a) Phase image taken at $f=0.5 \mathrm{~Hz}$

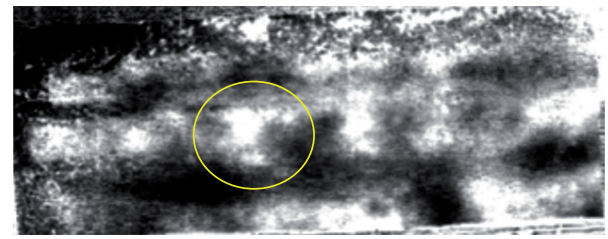

(b) Phase image taken at $f=0.04 \mathrm{~Hz}$

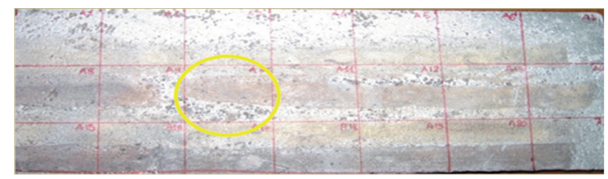

(c) Photo of the sample

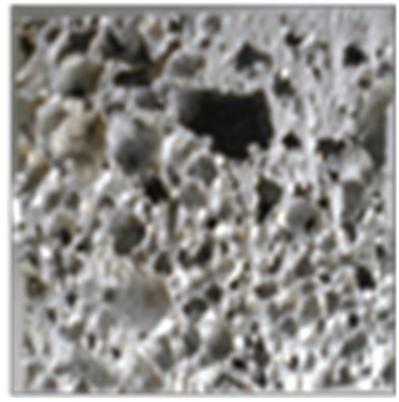

(d) Cut view of the zone encircled in (c)

FIGURE 9: A big pore detected through a phase image (b) with its location on the sample (c) and confirmation through cut view (d).

\section{Conclusions}

The use of infrared thermography for the evaluation of porosity in both a monolithic carbon/epoxy composite and an aluminum foam has been analyzed. More specifically, several different carbon/epoxy coupons were fabricated with enclosed each a very thin slag inclusion, to simulate either a delamination, or a backing film inclusion, and with a different amount of porosity. The obtained results show the following.

(i) The use of pulse thermography with the flash method allows mapping the thermal diffusivity and in turn gaining information about porosity amount and distribution. This is feasible through a correlation between the variation of thermal diffusivity and variation of porosity percentage. The variation of these two quantities is herein linked through the ratio $\psi$, which is proposed also as parameter to assess the effectiveness of this method. (ii) Flash thermography is also suitable to detect the presence of a very thin slag inclusion since the latter causes a local decrease of the thermal diffusivity.

(iii) Lock-in thermography allows for nondestructive evaluation of metal foams. It has been demonstrated the capability of LT to supply information about the distribution of pores and of macropores. However, more tests are necessary to completely assess the testing procedure for evaluation of porosity in metal foam cores, as well as in the complete sandwich structure.

As a general remark, the obtained results prove that infrared thermography may be used to gain two types of information. One regards the material characteristics (porosity amount, porosity distribution, and presence of defects). The second information regards the improvement of the manufacturing process through the evaluation of the output. This may be simply achieved, using the most adequate infrared imaging device and test procedure, in a fast and safe (for the component and the personnel) way.

\section{Acknowledgment}

Metal foam specimens were supplied by Professor L. Carrino and Professor M. Durante.

\section{References}

[1] A. A. Baker, S. Dutton, and D. Kelly, Composite Materials for Aircraft Structures, AIAA Education Series, 2nd edition, 2004.

[2] A. C. Loos and G. S. Springer, "Calculation of cure process variables during cure of graphite-epoxy composites," in Composite Materials, Quality Assurance and Processing, C. E. Brownin, Ed., vol. 797 of ASTM STP, pp. 110-118, 1983.

[3] L. Liu, B. M. Zhang, D. F. Wang, and Z. J. Wu, "Effects of cure cycles on void content and mechanical properties of composite laminates," Composite Structures, vol. 73, no. 3, pp. 303-309, 2006.

[4] F. Y. C. Boey and S. W. Lye, "Void reduction in autoclave processing of thermoset composites: part 1: high pressure effects on void reduction," Composites, vol. 23, no. 4, pp. 261-265, 1992.

[5] E. A. Birt and R. A. Smith, "A review of NDE methods for porosity measurement in fibre-reinforced polymer composites," Insight, vol. 46, no. 11, pp. 681-686, 2004.

[6] J. Schuller and R. Oster, "Classification of porosity by ultrasonic in carbon fibre helicopter structures based on micro computed tomography," in Proceedings of the European Conference on Nondestructive Testing, Berlin, Germany, September 2006.

[7] J.-W. Park, D. J. Kim, K.-H. Im et al., "Ultrasonic influence of porosity level on CFRP Composite laminates using rayleigh probe waves," Acta Mechanica Solida Sinica, vol. 21, no. 4, pp. 298-307, 2008.

[8] L. Lin, M. Luo, H. T. Tian, X. M. Li, and G. P. Guo, "Experimental investigation on porosity of carbon fibre-reinforced composite using ultrasonic attenuation coefficient," in Proceedings of the World Conference on Nondestructive Testing, Shanghai, China, October 2008.

[9] I. M. Daniel, S. C. Wooh, and I. Komsky, "Quantitative porosity characterization of composite materials by means of ultrasonic 
attenuation measurements," Journal of Nondestructive Evaluation, vol. 11, no. 1, pp. 1-8, 1992.

[10] EN 2564. Carbon fibre laminates-Determination of the fibre-, resin- and void contents, 1998.

[11] G. Hendorfer, G. Mayr, G. Zauner, M. Haslhofer, and R. Pree, "Quantitative determination of porosity by active thermography," vol. 26, pp. 702-708.

[12] J. Kastner, B. Plank, D. Salaberger, and J. Sekelja, "Defect and porosity determination of fibre reinforced polymers by X-ray computed tomography," in Proceedings of the 2nd International Symposium on NDT in Aerospace, Hamburg, Germany, November 2010.

[13] G. Mayr and G. Handorfer, "Porosity Determination by pulsed Thermography in reflection mode," in Proceedings of the 10th International Conference on Quantitative InfraRed Thermography, Quebec, Canada, July, 2010.

[14] J. Banhart, "Manufacture, characterisation and application of cellular metals and metal foams," Progress in Materials Science, vol. 46, no. 6, pp. 559-632, 2001.

[15] D. T. Queheillalt, D. J. Sypeck, and H. N. G. Wadley, "Ultrasonic characterization of cellular metal structures," Materials Science and Engineering A, vol. 323, no. 1-2, pp. 138-147, 2002.

[16] L. P. Lefebvre, A. Blouin, S. M. Rochon, and M. N. Bureau, "Elastic response of titanium foams during compression tests and using laser-ultrasonic probing," Advanced Engineering Materials, vol. 8, no. 9, pp. 841-846, 2006.

[17] G. M. Carlomagno, L. Carrino, M. Durante, S. Franchitti, and C. Meola, "Density evaluation in metal foams with lock-in thermography," in Proceedings of the 10th A.I.TE.M. Conference Enhancing the Science of Manufacturing, pp. 1-10, 2011.

[18] C. Meola and G. M. Carlomagno, "Infrared thermography in non-destructive inspection: theory and practice," in Recent Advances in Non Destructive Inspection, C. Meola, Ed., pp. 89123, Nova Science, New York, NY, USA, 2010.

[19] C. Meola and C. Toscano, "NonDestructive evaluation of carbon fiber reinforced polymers with ultrasonics and infrared thermography: an overview on historical steps and patents," Recent Patents on Materials Science, vol. 5, pp. 48-67, 2012.

[20] C. Meola and G. M. Carlomagno, "Recent advances in the use of infrared thermography," Measurement Science and Technology, vol. 15, no. 9, pp. R27-R58, 2004.

[21] C. Meola and G. M. Carlomagno, "Application of infrared thermography to adhesion science," Journal of Adhesion Science and Technology, vol. 20, no. 7, pp. 589-632, 2006.

[22] G. M. Carlomagno and P. G. Berardi, "Unsteady thermotopography in non-Destructive Testing," in III Infrared Information Exchange, C. Warren, Ed., pp. 33-40, St. Louis, Mo, USA, 1976.

[23] J. L. Beaudoin, E. Merienne, R. Danjoux, and M. Egee, "Numerical system for infrared scanners and application to the subsurface control of materials by photothermal radiometry," in Proceedings of the Infrared Technology and Applications, vol. 590 of Proceeding of SPIE, pp. 287-292, November 1985.

[24] P. K. Kuo, Z. J. Feng, T. Ahmed, L. D. Favro, R. L. Thomas, and J. Hartikainen, "Parallel thermal wave imaging using a vector lockin video technique," in Photoacoustic and Photothermal Phenomena, P. Hess and J. Pelzl, Eds., pp. 415-418, Springer, Heidelberg, Germany, 1987.

[25] G Busse, D. Wu, and W. Karpen, "Thermal wave imaging with phase sensitive modulated thermography," Journal of Applied Physics, vol. 71, pp. 3962-3965, 1992.
[26] A. Lehto, J. Jaarinen, T. Tiusanen, M. Jokinen, and M. Luukkala, "Magnitude and phase in thermal wave imaging," Electronics Letters, vol. 17, no. 11, pp. 364-365, 1981.

[27] C. A. Bennett Jr. and R. R. Patty, “Thermal wave interferometry: a potential application of the photoacoustic effect," Applied Optics, vol. 21, no. 1, pp. 49-54, 1981.

[28] G. Busse, "Optoacustic phase angle measurement for probing a metal," Applied Physics Letters, vol. 35, pp. 759-760, 1979.

[29] R. L. Thomas, J. J. Pouch, Y. H. Wong, L. D. Favro, P. K. Kuo, and A. Rosencwaig, "Subsurface flaw detection in metals by photoacoustic microscopya," Journal of Applied Physics, vol. 51, no. 2, pp. 1152-1156, 1980.

[30] G. Busse, "Optoacoustic and photothermal material inspection techniques," Applied Optics, vol. 21, pp. 107-110, 1982.

[31] C Toscano and C. Vitiello, "Study of the influence of the staking sequence on porosity formation in carbon fibre composites," Journal of Applied Polymer Science, vol. 122, no. 6, pp. 3583-3589, 2011.

[32] W. J. Parker, R. J. Jenkins, C. P. Butler, and G. L. Abbott, “Flash method of determining thermal diffusivity, heat capacity, and thermal conductivity," Journal of Applied Physics, vol. 32, no. 9, pp. 1679-1684, 1961.

[33] C. Toscano, C. Meola, M. C. Iorio, and G. M. Carlomagno, "Porosity and inclusion detection in CFRP by infrared thermography," Advances in Optical Technologies, vol. 2012, Article ID 765953, 6 pages, 2012. 

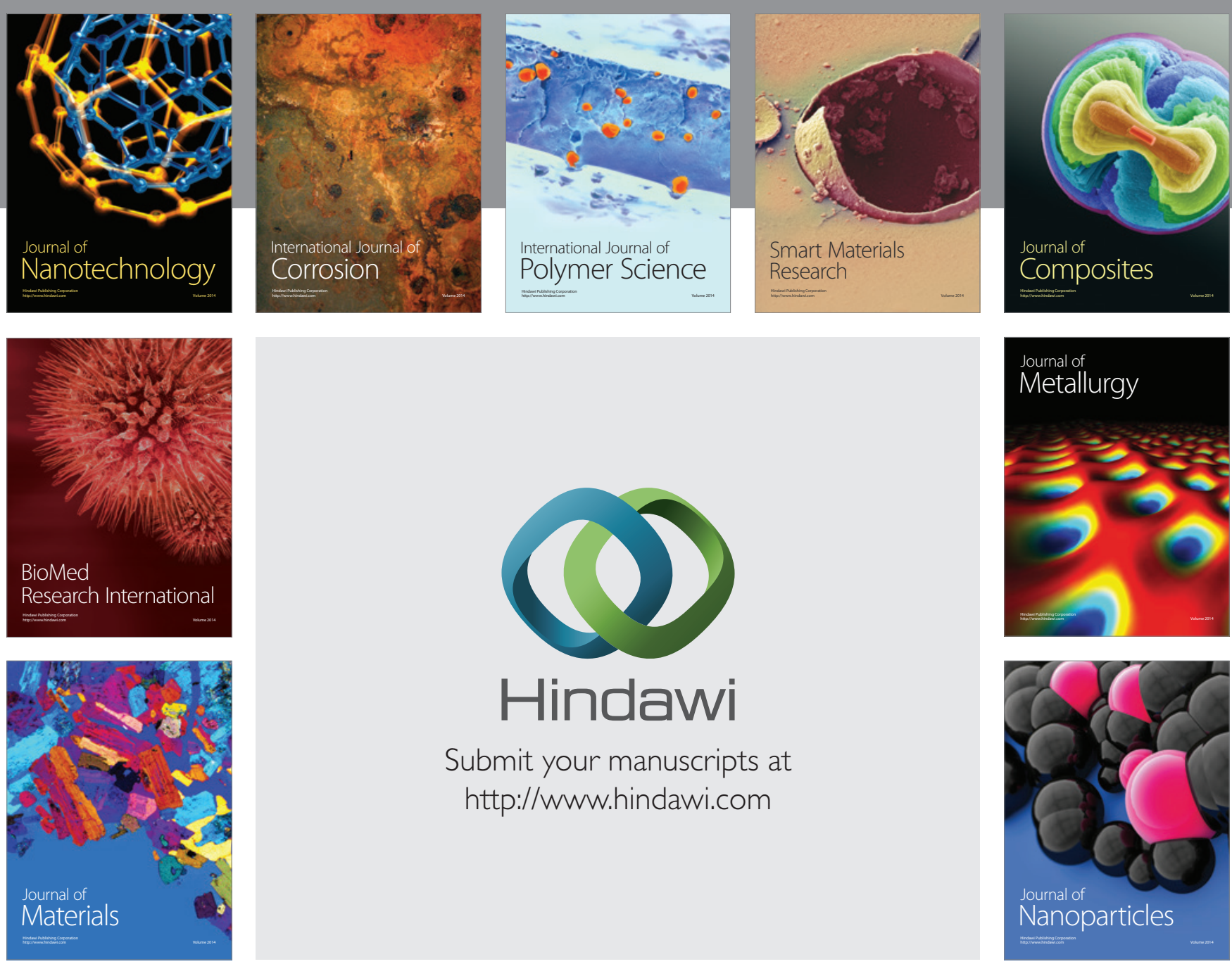

Submit your manuscripts at http://www.hindawi.com
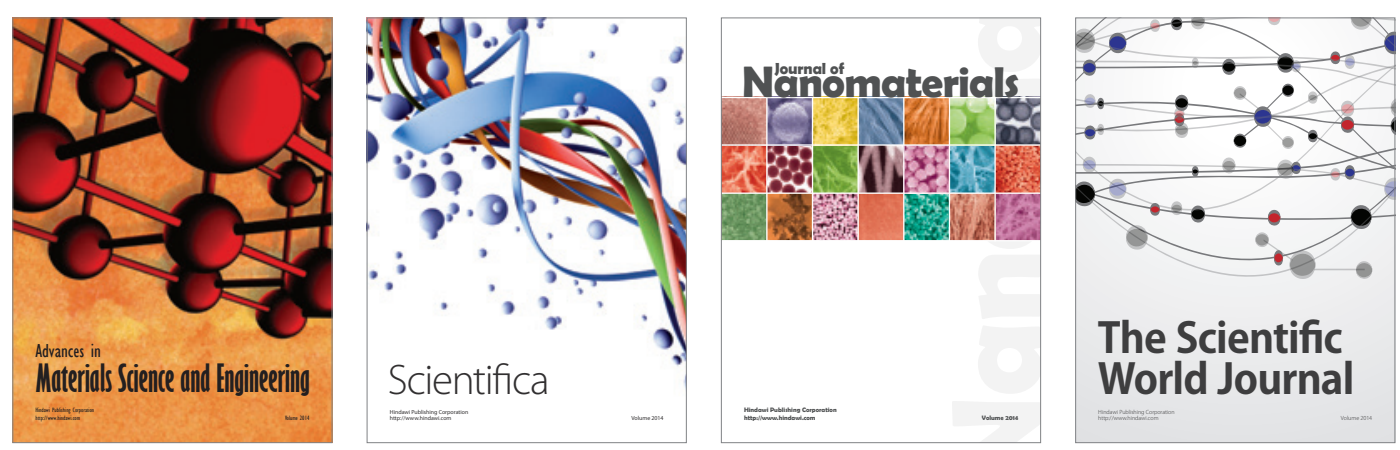

\section{The Scientific World Journal}
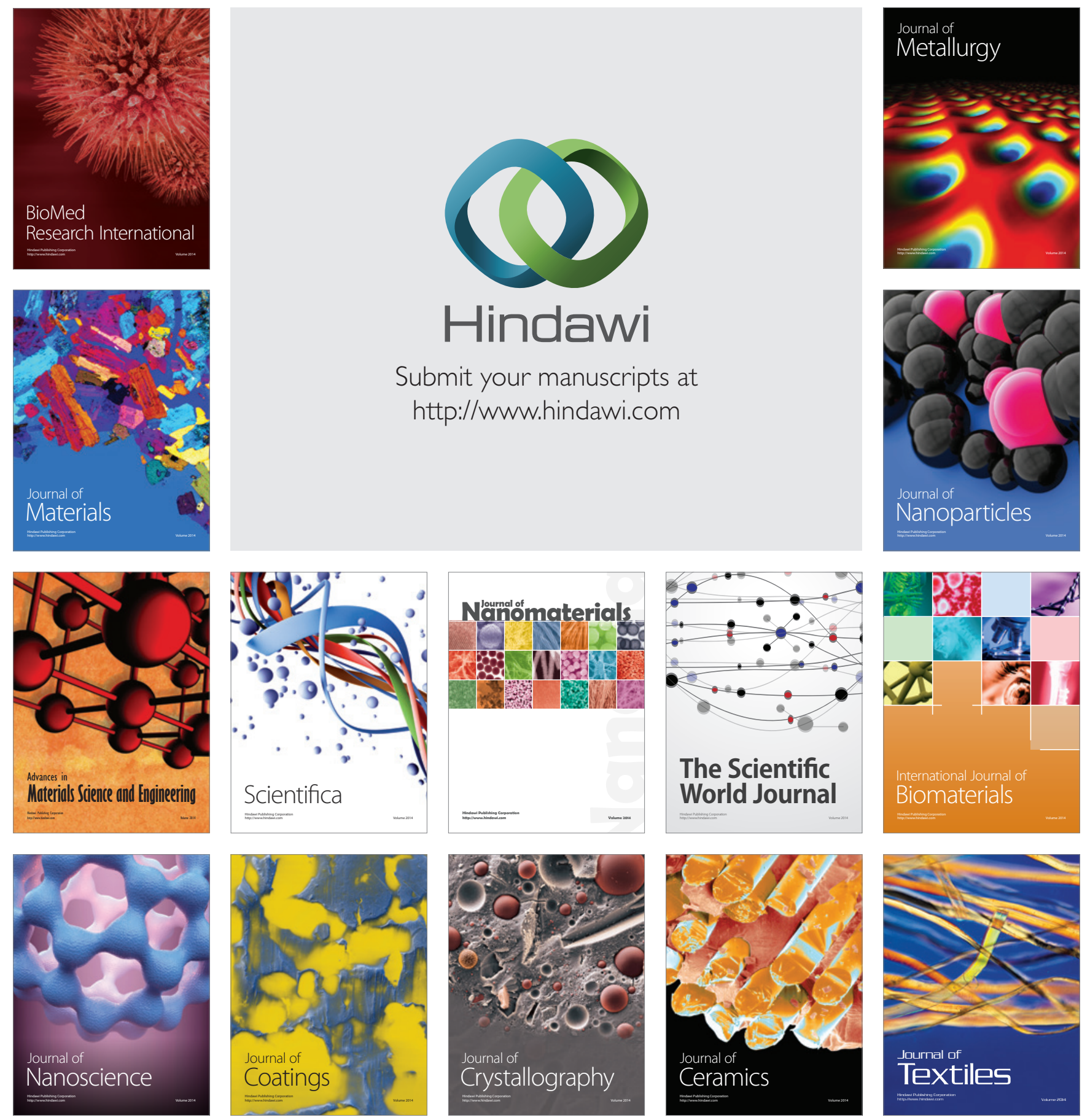